\title{
ERRATA, VOLUME 72
}

R. J. Warne, On certain bisimple inverse semigroups, pp. 679-682.

Page 680 , Last line of Theorem 3: Replace " $\bar{g}$ " by " $\bar{g} \theta^{-i}$ " and " $i \in I$ " by " $i \in I$ and $i<0$."

R. S. Freeman, On the spectrum and resolvent of homogeneous elliptic differential operators with constant coefficients, pp. 538-541.

The remark following Lemma 7 is not correct and as a consequence all the statements which follow are incorrect. Moreover, the statement of Theorem 6 contains an obvious omission. Correct statements appear below:

ThEOREM. A necessary and sufficient condition that the map

$$
u \rightarrow\left\{(A-\lambda) u, B_{0} u, \cdots, B_{m-1} u\right\}
$$

be a topological isomorphism of $H^{2 m}(\Omega)$ onto $H^{0}(\Omega) \times \prod_{j=0}^{m-1} H^{2 m-m_{j}-1 / 2}(\Gamma$ is that all of the following be true

$1^{\circ}$. $(A, B)$ is closable elliptic;

$2^{\circ}$. $A(\xi)-\lambda \neq 0$ for $\xi \in I R^{n}$;

$3^{\circ}$. The polynomials $\left\{B_{j}\right\}$ are linearly independent modulo $A_{\lambda}^{+}$.

This should replace Theorems 6 and 8 of the original paper. Moreover, condition $3^{\circ}$ must be added to Theorems 11 and 12 .

Three lines down from Definition 1 , in the definition of $c$, max should be replaced by min.

REMARK $1^{\circ}$. The proof outlined for Lemma 3 is unnecessarily complicated. It is a simple consequence of Rouche's Theorem.

$2^{\circ}$. The homogeneity requirements for $A$ and the $B_{j}$ 's are unnecessary. The results have been obtained without them by the present writer and independently by Schechter.

E. F. Assmus, Jr. and H. F. Mattson, Jr., Disjoint Steiner systems associated with the Mathieu groups, pp. 843-846.

Add: "This research was partly supported by the U. S. Air Force under Contract AF-19-(628)-5998." 